\title{
BER Performance Analysis of Cooperative DaF Relay Networks and a New Optimal DaF Strategy
}

\author{
George A. Ropokis, Athanasios A. Rontogiannis, Member, IEEE, and Kostas Berberidis, Senior Member, IEEE
}

\begin{abstract}
An analytical bit error rate (BER) performance study of three detect-and-forward (DaF) policies under a common framework is presented in this paper. More specifically, the direct, thresholded [1] and link-adaptive [2] schemes are studied, which differ in the way the decision on the transmitted symbol is forwarded from the relay to the destination. The analysis is carried out for a single relay DaF transmission protocol and takes into account the symbol decision errors that may occur at the relay. Simple closed-form analytical BER expressions are derived for all three schemes, which are completely verified by simulations. Moreover, by minimizing a properly selected conditional BER expression, a novel optimal DaF strategy is proposed, which outperforms all previously known related methods.
\end{abstract}

Index Terms-Cooperative networks, regenerative relays, detect-and-forward, BER performance.

\section{INTRODUCTION}

$\mathbf{T}$ HE design of cooperative diversity communication schemes and the analysis of their performance have attracted considerable attention recently. In relevant literature, most works are devoted to cooperative systems employing non-regenerative relays, i.e., using an amplify-and-forward (AaF) strategy. This is mainly due to the fact that $\mathrm{AaF}$ schemes offer full diversity gains for large values of signalto-noise ratio (SNR) [3], [4]. Nevertheless, their practical implementation requires expensive RF chains [5], as opposed to detect-and-forward (DaF) transmission schemes, in which the relays regenerate the received signals with only simple additional digital processing. In [6] a DaF cooperative method is described based on a distributed version of the well known Alamouti code and a suboptimum, as compared to the maximum likelihood (ML), receiver is proposed. In [7] and [5] two different maximal ratio combining (MRC) based cooperative schemes are developed and suboptimum, low complexity detection criteria are proposed. For all the above schemes, derivation of analytical closed-form expressions for the bit error rate (BER) is rather complicated. Recently in [1], a detailed BER analysis of a single relay DaF network has been presented for BPSK signaling, MRC at the destinationreceiver, and threshold-based retransmission at the relay. Four

Manuscript received July 26, 2010; revised December 12, 2010; accepted January 13, 2010. The associate editor coordinating the review of this letter and approving it for publication was C.-C. Chong.

G. A. Ropokis and A. A. Rontogiannis are with the Institute for Space Applications and Remote Sensing, National Observatory of Athens, 15236, Palea Penteli, Athens, Greece (e-mail: \{ropokis, tronto\}@space.noa.gr).

K. Berberidis is with the Department of Computer Engineering \& Informatics, University of Patras 26500, Rio-Patras, Greece (email:bebrerid@ceid.upatras.gr).

This work was supported in part by the FP6 project Cooperative and Opportunistic Communications in Wireless Networks (COOPCOM, FP6033533).

Digital Object Identifier 10.1109/TWC.2011.020811.101344

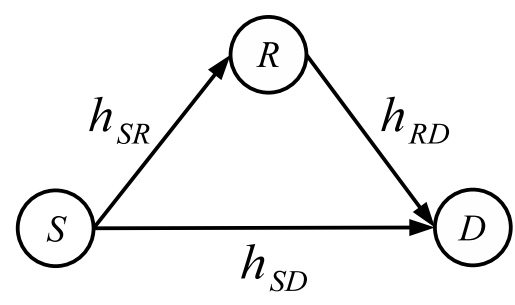

Fig. 1. Block diagram of a single-relay network.

different scenaria have been considered in [1], depending on whether instantaneous or average SNR information of the various channels, is available at the relay. For all four scenaria, the optimum thresholds have been derived, that result in the minimum end-to-end BER performance.

In this paper, we consider the first scenario of [1] and analyze the BER performance of three different DaF schemes, namely simple MRC, thresholded MRC with a fixed threshold and link adaptive MRC [2]. A unified analysis framework is developed and a novel approach is utilized based on the manipulation of the decision variable at the destination as a quadratic form in normal random variables. Analytical closedform BER expressions are derived for all three schemes, which are completely verified via simulations. Moreover, by minimizing a properly selected conditional bit error probability at the destination, the optimal weighting of the symbolsdecisions forwarded by the relay is derived. As verified by simulations, the proposed method outperforms all previously known schemes in terms of BER for all SNR values.

The rest of the paper is organized as follows. In Section II, the system model adopted in the paper is presented and three cooperative strategies are defined in a unified way. BER performance analysis of these cooperative schemes is presented in Section III. A new minimum BER combining scheme is proposed in Section IV. Simulation results are given in Section $\mathrm{V}$ and the basic conclusions of this work are summarized in Section VI.

\section{System Model and Problem Formulation}

The basic structure of the communication network studied in this paper is shown in Fig. 1. We assume that a single BPSK symbol $s$ with unit energy $(s= \pm 1)$ is transmitted in two consecutive time slots. During the first time slot, the source $(S)$ transmits symbol $s$, which is received by both the relay $(R)$ and the destination $(D)$. As a result, the signal received at the destination at this time slot is

$$
y_{S D}=\sqrt{P_{S}} h_{S D} s+n_{S D} .
$$

$P_{S}$ is the power transmitted by $S$, assuming a bandwidth of $1 \mathrm{~Hz}$, and $n_{S D}$ stands for the complex additive white 
Gaussian noise (AWGN) on the $S \rightarrow D$ channel, whose real and imaginary parts have variance equal to $\sigma_{D}^{2} / 2$. Assuming Rayleigh fading, the channel $h_{S D}$, is also a zero mean complex Gaussian random variable $(\mathrm{RV})$ with variance $\sigma_{S D}^{2}$. Hence, by defining the SNR of the $S \rightarrow D$ channel as

$$
\gamma_{S D}=\frac{P_{S}\left|h_{S D}\right|^{2}}{\sigma_{D}^{2}}
$$

the probability density function (PDF) of $\gamma_{S D}$ can be expressed as [8]

$$
p_{\gamma_{S D}}(\gamma)=\frac{\exp \left(-\frac{\gamma}{\bar{\gamma}_{S D}}\right)}{\bar{\gamma}_{S D}} \text { with } \quad \bar{\gamma}_{S D}=\frac{P_{S} \sigma_{S D}^{2}}{\sigma_{D}^{2}} .
$$

The signal received at the relay in the same time slot is written as

$$
y_{S R}=\sqrt{P_{S}} h_{S R} s+n_{S R}
$$

where $\left|h_{S R}\right|$ is a Rayleigh $\mathrm{RV}$, that is, $h_{S R}$ is zero mean complex Gaussian distributed with variance $\sigma_{S R}^{2}$ and $n_{S R}$ is complex AWGN with variance $\sigma_{R}^{2}$. Therefore, the PDF of the SNR at the relay will be given by

$$
p_{\gamma_{S R}}(\gamma)=\frac{\exp \left(-\frac{\gamma}{\bar{\gamma}_{S R}}\right)}{\bar{\gamma}_{S R}} \quad \text { with } \quad \bar{\gamma}_{S R}=\frac{P_{S} \sigma_{S R}^{2}}{\sigma_{R}^{2}} .
$$

At the relay, a decision $\tilde{s}$ for the transmitted symbol $s$ is taken. A weighted version of this decision is transmitted to the destination during the second time slot, while the source remains idle. Hence, the signal received at the destination in the second time slot is written as

$$
y_{R D}=\sqrt{w P_{R}} h_{R D} \tilde{s}+n_{R D}
$$

where $P_{R}$ is the power factor at the relay and $w$ is a weighting factor quantifying the reliability of the decision at the relay. The channel $h_{R D}$ and the noise $n_{R D}$ are also assumed zero mean complex Gaussian RVs with variances $\sigma_{R D}^{2}$ and $\sigma_{D}^{2}$, respectively. The PDF of $\gamma_{R D}$ given the weighting factor $w$ is then written as

$$
p_{\gamma_{R D} \mid w}(\gamma)=\frac{\exp \left(-\frac{\gamma}{w \bar{\gamma}_{R D}}\right)}{w \bar{\gamma}_{R D}} \quad \text { with } \quad \bar{\gamma}_{R D}=\frac{P_{R} \sigma_{R D}^{2}}{\sigma_{D}^{2}} .
$$

For the transmission scheme described above, a maximum likelihood (ML) detection at the destination would require knowledge of the instantaneous probability of error at the relay [7]. Such a requirement renders the practical implementation of the ML detector extremely complex. In this work, as in [1], we consider a suboptimum receiver defined as

$$
\hat{s}=\operatorname{sign}\{d\}
$$

where the decision variable $d$ is the output of a maximal ratio combiner (MRC), which combines at the destination the signals received by the source and the relay as follows

$$
\begin{aligned}
d & =\Re\left\{\sqrt{P_{S}} h_{S D}^{*} y_{S D}+\sqrt{w P_{R}} h_{R D}^{*} y_{R D}\right\} \\
& =\Re\left\{P_{S}\left|h_{S D}\right|^{2} s+\sqrt{P_{S}} h_{S D}^{*} n_{S D}\right. \\
& \left.+w P_{R}\left|h_{R D}\right|^{2} \tilde{s}+\sqrt{w P_{R}} h_{R D}^{*} n_{R D}\right\}
\end{aligned}
$$

where $\Re\{x\}$ stands for the real part of $x$.

In the analysis presented below, the following three relay strategies are studied, which differ in the way the weighting factort $w$ is chosen.

- Simple MRC: In this case $w$ is always chosen equal to 1. In other words, the relay always forwards its decisions, assuming that they are correct.

- Thresholded MRC: In thresholded MRC the relay is employed only if the condition $\gamma_{S R} \geq \gamma_{0}$ is satisfied, where $\gamma_{0}$ is a predefined threshold. That is, the weight $w$ is determined by the rule

$$
w= \begin{cases}0, & \text { if } \quad \gamma_{S R}<\gamma_{0} \\ 1, & \text { if } \quad \gamma_{S R} \geq \gamma_{0}\end{cases}
$$

- Link-adaptive MRC: In link-adaptive MRC the desicion at the relay is properly weighted depending on the relation between the instantaneous SNR of the $S \rightarrow R$ link and the average SNR of the $R \rightarrow D$ link [2]. More specifically, $w$ is selected according to the rule

$$
w= \begin{cases}\frac{\gamma_{S R}}{\bar{\gamma}_{R D},} & \text { if } \gamma_{S R}<\bar{\gamma}_{R D} \\ 1, & \text { if } \gamma_{S R} \geq \bar{\gamma}_{R D}\end{cases}
$$

Note that thresholded MRC has also been studied in [1]. While here a fixed threshold is considered, in [1] the emphasis has been given to the derivation of the optimum threshold, which minimizes the end-to-end BER. In the next section closedform BER expressions are derived for the above transmission strategies based on the theory of quadratic forms in normal RVs. Then a new approach is proposed for selecting the weight $w$, whose BER performance is superior compared to all previously presented methods.

\section{BER PERFORMANCE ANALYSIS}

In order to analyze the performance of the model presented in the previous section in terms of BER, the statistics of the decision variable $d$ and the weighting factor $w$ need to be studied. It can be noticed that in all previously presented cooperation strategies, $w$ is either a constant, or a RV related to the instantaneous SNR value of the $S \rightarrow R$ link, $\gamma_{S R}$, and the mean value of the SNR at the $R \rightarrow D$ link. Therefore, assuming an equiprobable source and a transmitted symbol $s=1$, the average BER is given by the following general expression

$$
\begin{aligned}
P_{e}= & \int_{0}^{\infty} P_{e \mid \gamma}^{R} \operatorname{Pr}(d<0 \mid s=1, \tilde{s}=-1, w(\gamma)) p_{\gamma_{S R}}(\gamma) d \gamma+ \\
& \int_{0}^{\infty}\left(1-P_{e \mid \gamma}^{R}\right) \operatorname{Pr}(d<0 \mid s=1, \tilde{s}=1, w(\gamma)) p_{\gamma_{S R}}(\gamma) d \gamma
\end{aligned}
$$

where $P_{e \mid \gamma}^{R}$ is the probability of error at the relay conditioned on the instantaneous SNR $\gamma_{S R}=\gamma$. This probability is given by $P_{e \mid \gamma}^{R}=Q(\sqrt{2 \gamma})$ [8], where $Q(\cdot)$ is the Q-function. By inspecting (12), it can be seen that in order to express the BER in closed form, the cumulative distribution function (CDF) of $d$ at zero conditioned on $s, \tilde{s}$ and $w$, is required. Let us start 
by expressing $d$ in the following analytical form

$$
\begin{aligned}
d & =\left(P_{S} s \Re\left\{h_{S D}\right\}+\sqrt{P_{S}} \Re\left\{n_{S D}\right\}\right) \Re\left\{h_{S D}\right\} \\
& +\left(P_{S} s \Im\left\{h_{S D}\right\}+\sqrt{P_{S}} \Im\left\{n_{S D}\right\}\right) \Im\left\{h_{S D}\right\} \\
& +\left(w P_{R} \tilde{s} \Re\left\{h_{R D}\right\}+\sqrt{w P_{R}} \Re\left\{n_{R D}\right\}\right) \Re\left\{h_{R D}\right\} \\
& +\left(w P_{R} \tilde{s} \Im\left\{h_{R D}\right\}+\sqrt{w P_{R}} \Im\left\{n_{R D}\right\}\right) \Im\left\{h_{R D}\right\} .
\end{aligned}
$$

where $\Im\{x\}$ stands for the imaginary part of $x$. Then, by defining the $8 \times 1$ Gaussian vector

$$
\begin{aligned}
\mathbf{v}= & {\left[P_{S} s \Re\left\{h_{S D}\right\}+\sqrt{P_{S}} \Re\left\{n_{S D}\right\}, \Re\left\{h_{S D}\right\},\right.} \\
& P_{S} s \Im\left\{h_{S D}\right\}+\sqrt{P_{S}} \Im\left\{n_{S D}\right\}, \Im\left\{h_{S D}\right\}, \\
& w P_{R} \tilde{s} \Re\left\{h_{R D}\right\}+\sqrt{w P_{R}} \Re\left\{n_{R D}\right\}, \Re\left\{h_{R D}\right\}, \\
& \left.w P_{R} \tilde{s} \Im\left\{h_{R D}\right\}+\sqrt{w P_{R}} \Im\left\{n_{R D}\right\}, \Im\left\{h_{R D}\right\}\right]
\end{aligned}
$$

the decision variable $d$ can equivalently be written as

$$
d=v_{1} v_{2}+v_{3} v_{4}+v_{5} v_{6}+v_{7} v_{8}
$$

where $v_{i}$ stands for the $i$-th element of vector $\mathbf{v}$.

Clearly, $\mathbf{v}$ has zero mean. Furthermore, it can be shown that its covariance matrix $\mathbf{C}_{\mathbf{v}}$ is expressed as

$$
\mathbf{C}_{\mathbf{v}}=\left[\begin{array}{cc}
\mathbf{C}\left(P_{S}, s, \sigma_{S D}^{2}, \sigma_{D}^{2}\right) & \mathbf{0}_{4 \times 4} \\
\mathbf{0}_{4 \times 4} & \mathbf{C}\left(w P_{R}, \tilde{s}, \sigma_{R D}^{2}, \sigma_{D}^{2}\right)
\end{array}\right]
$$

where $\mathbf{C}(\cdot, \cdot, \cdot, \cdot)$ is the parametric matrix

$$
\mathbf{C}(a, b, c, d)=\mathbf{I}_{2 \times 2} \otimes\left[\begin{array}{cc}
a^{2} b^{2} c+a d & a b c \\
a b c & c
\end{array}\right]
$$

with $\mathbf{I}_{m \times m}$ denoting the $m \times m$ identity matrix and $\otimes$ the Kronecker product operator. We can therefore write the decision variable as a quadratic form in normal RVs [9]

$$
d=\mathbf{v}^{T} \mathbf{A} \mathbf{v}
$$

where $\mathbf{A}$ is a symmetric matrix given by ${ }^{1}$

$$
\mathbf{A}=\mathbf{I}_{4 \times 4} \otimes\left[\begin{array}{cc}
0 & 1 / 2 \\
1 / 2 & 0
\end{array}\right] .
$$

Using the representation of $d$ as a quadratic form in normal RVs, as in (18), it is shown in the Appendix that the CDF of $d$ at zero for $s=1$ and $\tilde{s}= \pm 1$ is written as in Eqs. (20) and (21) given at the top of next page. Based on (20) and (21) the error probability for the three $\mathrm{DaF}$ schemes presented in the previous section, can be obtained in closed form, as explained below.

Simple MRC: Since for simple MRC $w$ is always independent of $\gamma_{S R}$ and equal to 1, (12) can be expressed as

$$
\begin{aligned}
P_{e} & =\left(\int_{0}^{\infty} P_{e \mid \gamma}^{R} p_{\gamma_{S R}}(\gamma) d \gamma\right) \\
& \times \operatorname{Pr}(d \leq 0 \mid s=1, \tilde{s}=-1, w=1) \\
& +\left(\int_{0}^{\infty}\left(1-P_{e \mid \gamma}^{R}\right) p_{\gamma_{S R}}(\gamma) d \gamma\right) \\
& \times \operatorname{Pr}(d \leq 0 \mid s=1, \tilde{s}=1, w=1)
\end{aligned}
$$

\footnotetext{
${ }^{1}$ Note that matrix $\mathbf{A}$ as given in (19) is the only symmetric matrix that satifies (18).
}

The average BER $P_{e}$ in (22) is written in a simple analytical formula by employing (20), (21) with $w=1$ and the following [8]

$$
\begin{aligned}
P_{e, \gamma_{S R}}^{R} & =\int_{0}^{\infty} P_{e \mid \gamma}^{R} p_{\gamma_{S R}}(\gamma) d \gamma \\
& =\frac{1}{\bar{\gamma}_{S R}} \int_{0}^{\infty} Q(\sqrt{2 \gamma}) \exp \left(-\frac{\gamma}{\bar{\gamma}_{S R}}\right) d \gamma \\
& =\frac{1}{2}\left(1-\sqrt{\frac{\bar{\gamma}_{S R}}{1+\bar{\gamma}_{S R}}}\right)
\end{aligned}
$$

Thresholded MRC: In the case of thresholded MRC, (12) takes the form

$$
\begin{aligned}
P_{e} & =\operatorname{Pr}\left(\gamma_{S R}<\gamma_{0}\right) \operatorname{Pr}(d \leq 0) \\
& +P_{e, \gamma_{S R} \geq \gamma_{0}}^{R} \operatorname{Pr}(d \leq 0 \mid s=1, \tilde{s}=-1, w=1) \\
& +\left(\operatorname{Pr}\left(\gamma_{S R} \geq \gamma_{0}\right)-P_{e, \gamma_{S R} \geq \gamma_{0}}^{R}\right) \\
& \times \operatorname{Pr}(d \leq 0 \mid s=1, \tilde{s}=1, w=1) .
\end{aligned}
$$

Note that the first term in the RHS of (24) is not related to the probability of error at the relay, because the relay is not employed for $\gamma_{S R}<\gamma_{0}$. In the same term, the probability of error at $D, \operatorname{Pr}(d \leq 0)$, can be computed as the probability of error of the $S \rightarrow D$ link, i.e.,

$$
\begin{aligned}
\operatorname{Pr}(d \leq 0) & =\int_{0}^{\infty} Q(\sqrt{2 \gamma}) p_{\gamma_{S D}}(\gamma) d \gamma \\
& =\frac{1}{2}\left(1-\sqrt{\frac{\bar{\gamma}_{S D}}{1+\bar{\gamma}_{S D}}}\right) .
\end{aligned}
$$

Moreover, for $\gamma_{S R} \geq \gamma_{0}$, the probability of error at the relay $P_{e, \gamma_{S R} \geq \gamma_{0}}^{R}$ can be expressed as

$$
\begin{aligned}
P_{e, \gamma_{S R} \geq \gamma_{0}}^{R} & =\frac{1}{\bar{\gamma}_{S R}} \int_{\gamma_{0}}^{\infty} Q(\sqrt{2 \gamma}) \exp \left(-\frac{\gamma}{\bar{\gamma}_{S R}}\right) d \gamma \\
& =\frac{1}{2}\left(1-\sqrt{\frac{\bar{\gamma}_{S R}}{1+\bar{\gamma}_{S R}}}\right)-\mathcal{I}
\end{aligned}
$$

where $\mathcal{I}$ is given by

$$
\begin{aligned}
\mathcal{I} & =\frac{1}{2}-\frac{\operatorname{erfc}\left(\sqrt{\gamma_{0}}\right) \exp \left(-\frac{\gamma_{0}}{\bar{\gamma}_{S R}}\right)}{2} \\
& -\frac{1}{2} \sqrt{\frac{\bar{\gamma}_{S R}}{\bar{\gamma}_{S R}+1}} \operatorname{erf}\left(\sqrt{\gamma_{0} \frac{\bar{\gamma}_{S R}+1}{\bar{\gamma}_{S R}}}\right)
\end{aligned}
$$

and $\operatorname{erf}(x), \operatorname{erfc}(x)$ are the error function and the complementary error function, respectively. By substituting (20), (21), (25) and (26) in (24) we get a simple closed form BER expression for thresholded MRC. Note that here the desired expression for the BER is an exact one, whereas in [1] the respective expression is approximate.

Link-adaptive MRC: For this cooperative scheme, it is easy to see that the BER in (12) is given by Eq. (28) shown at the top of next page. The two integrals in the RHS of (28) can be easily evaluated using Gauss quadrature rules, while the last two terms can be effectively computed based on the relevant expressions derived in the previous paragraph for thresholded MRC. 


$$
\begin{array}{r}
\operatorname{Pr}(d \leq 0 \mid s=1, \tilde{s}=1, w)=\frac{\bar{\gamma}_{S D}}{2\left(\bar{\gamma}_{S D}-w \bar{\gamma}_{R D}\right)}\left(1-\sqrt{\frac{\bar{\gamma}_{S D}}{1+\bar{\gamma}_{S D}}}\right)-\frac{w \bar{\gamma}_{R D}}{2\left(\bar{\gamma}_{S D}-w \bar{\gamma}_{R D}\right)}\left(1-\sqrt{\frac{w \bar{\gamma}_{R D}}{1+w \bar{\gamma}_{R D}}}\right) \\
\operatorname{Pr}(d \leq 0 \mid s=1, \tilde{s}=-1, w)=\frac{\bar{\gamma}_{S D}\left(2 \bar{\gamma}_{S D}+1-2 \sqrt{\bar{\gamma}_{S D}^{2}+\bar{\gamma}_{S D}}\right)}{2\left(\bar{\gamma}_{S D}+w \bar{\gamma}_{R D}\right)\left(2 \bar{\gamma}_{S D}+1-2 \sqrt{\bar{\gamma}_{S D}^{2}+\bar{\gamma}_{S D}}\right)-4 w \bar{\gamma}_{R D}}\left(1-\sqrt{\frac{\bar{\gamma}_{S D}}{1+\bar{\gamma}_{S D}}}\right) \\
+\frac{w \bar{\gamma}_{R D}\left(2 w \bar{\gamma}_{R D}+1+2 \sqrt{w^{2} \bar{\gamma}_{R D}^{2}+w \bar{\gamma}_{R D}}\right)}{2\left(\bar{\gamma}_{S D}+w \bar{\gamma}_{R D}\right)\left(2 w \bar{\gamma}_{R D}+1+2 \sqrt{w^{2} \bar{\gamma}_{R D}^{2}+w \bar{\gamma}_{R D}}\right)-4 \bar{\gamma}_{S D}}\left(1+\sqrt{\frac{w \bar{\gamma}_{R D}}{1+w \bar{\gamma}_{R D}}}\right)
\end{array}
$$

$$
\begin{aligned}
P_{e} & =\int_{0}^{\bar{\gamma}_{R D}} P_{e \mid \gamma}^{R} p_{\gamma_{S R}}(\gamma) \operatorname{Pr}\left(d \leq 0 \mid s=1, \tilde{s}=-1, w=\gamma / \bar{\gamma}_{R D}\right) d \gamma \\
& +\int_{0}^{\bar{\gamma}_{R D}}\left(1-P_{e \mid \gamma}^{R}\right) \operatorname{Pr}\left(d \leq 0 \mid s=1, \tilde{s}=1, w=\gamma / \bar{\gamma}_{R D}\right) p_{\gamma_{S R}}(\gamma) d \gamma \\
& +P_{e, \gamma_{S R} \geq \bar{\gamma}_{R D}}^{R} \operatorname{Pr}(d \leq 0 \mid s=1, \tilde{s}=-1, w=1)+\left(\operatorname{Pr}\left(\gamma_{S R} \geq \bar{\gamma}_{R D}\right)-P_{e, \gamma_{S R} \geq \bar{\gamma}_{R D}}^{R}\right) \operatorname{Pr}(d \leq 0 \mid s=1, \tilde{s}=1, w=1)
\end{aligned}
$$

\section{A. Higher-Order Modulation Schemes}

Although the above performance analysis results apply for BPSK, a similar analysis can be carried out for higher-order modulation schemes such as Pulse Amplitude Modulation (PAM) and Quadrature Amplitude Modulation (QAM). For that purpose, first the detector for PAM and QAM needs to be defined. Building upon the form of the MRC receiver described in [1] and [2], this detector decides in favor of the symbol $\hat{s}$ that satisfies the equation

$$
\begin{aligned}
\hat{s} & =\underset{\hat{s} \in S}{\arg \min } \| \sqrt{w P_{R}} h_{R D}^{*} y_{R D}+\sqrt{P_{S}} h_{S D}^{*} y_{S D} \\
& -\left(w P_{R}\left|h_{R D}\right|^{2}+P_{S}\left|h_{S D}\right|^{2}\right) \hat{s} \|^{2}
\end{aligned}
$$

where $S$ denotes the symbols' alphabet.

With regard to PAM, the receiver in (29) is equivalent to a quantizer for the decision variable as expressed in (9). Specifically, assuming that symbol $s$ was transmitted, a detection error occurs, whenever

$$
\begin{aligned}
& d \leq\left(w P_{R}\left|h_{R D}\right|^{2}+P_{S}\left|h_{S D}\right|^{2}\right)\left(s-e_{0}\right) \text { or } \\
& d \geq\left(w P_{R}\left|h_{R D}\right|^{2}+P_{S}\left|h_{S D}\right|^{2}\right)\left(s+e_{0}\right)
\end{aligned}
$$

where $s-e_{0}$ and $s+e_{0}$ are the quantization points. From (30), the error probability for PAM is expressed as the probability that a quadratic form in normal RVs is smaller or larger than predefined thresholds. Hence, using again the statistics of quadratic forms, closed-form expressions for the symbol error rate (SER) and the BER of PAM can be derived.

As far as QAM is concerned, the detector in (29) can be implemented as two separate quantizers, one for the in-phase and the other for the quadrature signals respectively. As a consequence, the performance analysis procedure followed for PAM, can be also directly extended to QAM.

\section{Minimum BER Weight Selection}

In this section, by minimizing the BER at the destination conditioned on the instantaneous SNR at the relay, $\gamma_{S R}$, an optimal reliability factor $w$ is obtained. More specifically, this conditional BER is expressed as

$$
\begin{aligned}
P_{e \mid \gamma_{S R}}(w) & =P_{e \mid \gamma_{S R}}^{R} \operatorname{Pr}(d \leq 0 \mid s=1, \tilde{s}=-1, w) \\
& +\left(1-P_{e \mid \gamma_{S R}}^{R}\right) \operatorname{Pr}(d \leq 0 \mid s=1, \tilde{s}=1, w)
\end{aligned}
$$

where $P_{e \mid \gamma_{S R}}^{R}=Q\left(\sqrt{2 \gamma_{S R}}\right)$ and $\operatorname{Pr}(d \leq 0 \mid s=1, \tilde{s}=1, w)$, $\operatorname{Pr}(d \leq 0 \mid s=1, \tilde{s}=-1, w)$ are given by (20) and (21) respectively. Direct minimization of $P_{e \mid \gamma_{S R}}(w)$ in (31) is rather complicated and does not lead to a closed form solution for $w$. Thus, to simplify the expressions given in (20), and (21), which appear in (31) the first order Taylor series approximation of $\sqrt{x}$ around 1 has been utilized, i.e.,

$$
\sqrt{x} \approx 1+\frac{1}{2}(x-1) .
$$

It is easily verified that (32) is a very accurate approximation for the square roots of the form $\sqrt{a /(1+a)}$ appearing in (20) and (21). For square roots of the form $\sqrt{a^{2}+a}$, the following approximation has been used

$$
\begin{aligned}
\sqrt{a^{2}+a} & =(1+a) \sqrt{\frac{a}{1+a}} \\
& \approx(1+a)\left(1+\frac{1}{2}\left(\frac{a}{1+a}-1\right)\right)=\frac{1}{2}+a
\end{aligned}
$$

Based on these approximations the probabilities in (20) and (21) are written as

$$
\operatorname{Pr}(d \leq 0 \mid s=1, \tilde{s}=1, w) \approx \frac{1}{4\left(1+\bar{\gamma}_{S D}\right)\left(1+w \bar{\gamma}_{R D}\right)}
$$

and

$$
\begin{aligned}
\operatorname{Pr}(d & \leq 0 \mid s=1, \tilde{s}=-1, w) \\
& \approx \frac{\left(4 w \bar{\gamma}_{R D}+3\right)\left(1+2 w \bar{\gamma}_{R D}\right)}{4\left(1+w \bar{\gamma}_{R D}\right)\left(2 \bar{\gamma}_{S D}+2 w \bar{\gamma}_{R D}+1\right)} .
\end{aligned}
$$

By substituting (34) and (35) in (31) and setting $q=P_{e \mid \gamma_{S R}}^{R}$ we get (36). Taking the derivative of (36) and equating it to zero leads to the solutions for $w$ shown at the top of next page. The solution which minimizes $P_{e \mid \gamma_{S R}}(w)$ for $0 \leq w \leq 1$ is then used to weight the decision at the relay. If none 


$$
\begin{gathered}
P_{e \mid \gamma_{S R}}(w) \approx \frac{8 q\left(1+\bar{\gamma}_{S D}\right) w^{2} \bar{\gamma}_{R D}^{2}+\left(2+8 q+10 q \bar{\gamma}_{S D}\right) w \bar{\gamma}_{R D}+1+2 q+\bar{\gamma}_{S D}(2+q)}{4\left(1+\bar{\gamma}_{S D}\right)\left(2 w^{2} \bar{\gamma}_{R D}^{2}+\left(3+2 \bar{\gamma}_{S D}\right) w \bar{\gamma}_{R D}+\left(1+2 \bar{\gamma}_{S D}\right)\right)} \\
w_{ \pm}=\frac{-\left(q\left(2+11 \bar{\gamma}_{S D}+8 \bar{\gamma}_{S D}^{2}\right)-1-2 \bar{\gamma}_{S D}\right)}{2 \bar{\gamma}_{R D}\left(q\left(2+5 \bar{\gamma}_{S D}+4 \bar{\gamma}_{S D}^{2}\right)-1\right)} \pm \frac{\sqrt{2 q \bar{\gamma}_{S D}\left(1-5 \bar{\gamma}_{S D}+2 \bar{\gamma}_{S D}^{2}+8 \bar{\gamma}_{S D}^{3}-q\left(2-7 \bar{\gamma}_{S D}-5 \bar{\gamma}_{S D}^{2}+4 \bar{\gamma}_{S D}^{3}\right)\right)}}{2 \bar{\gamma}_{R D}\left(q\left(2+5 \bar{\gamma}_{S D}+4 \bar{\gamma}_{S D}^{2}\right)-1\right)}
\end{gathered}
$$

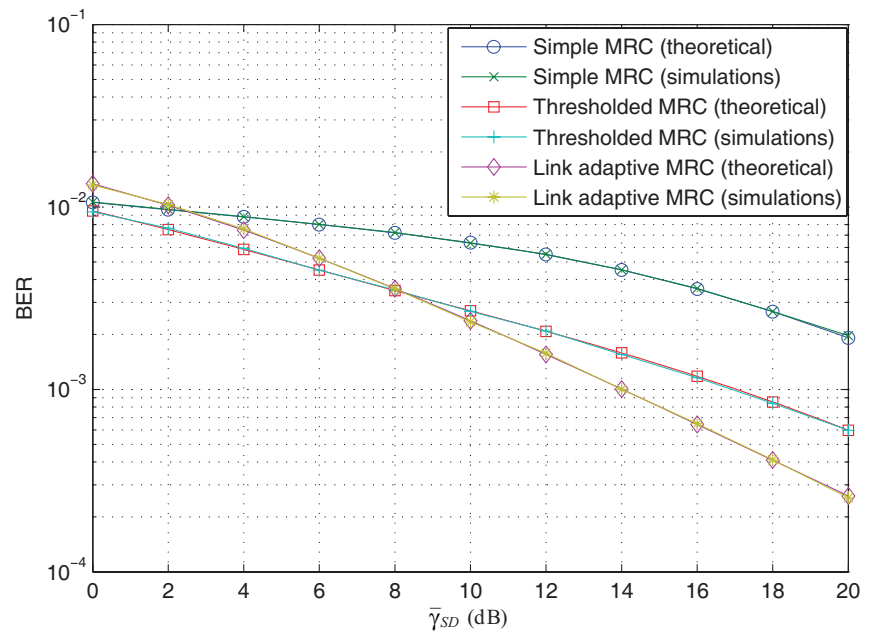

Fig. 2. Performance comparison of three cooperative diversity schemes for various $\bar{\gamma}_{S D}$ SNR values and $\bar{\gamma}_{S R}=\bar{\gamma}_{R D}=15 \mathrm{~dB}$. In thresholded MRC, $\gamma_{0}=0 \mathrm{~dB}$.

of the solutions are in the interval $[0,1]$, this means that $P_{e \mid \gamma_{S R}}(w)$ does not have any extrema in this interval. Thus, if $P_{e \mid \gamma_{S R}}(0)<P_{e \mid \gamma_{S R}}(1), P_{e \mid \gamma_{S R}}(0)$ will be the minimum value of $P_{e \mid \gamma_{S R}}(w)$ in $[0,1]$, and $w$ is set equal to 0 . Similarly, if $P_{e \mid \gamma_{S R}}(1)<P_{e \mid \gamma_{S R}}(0), w$ is set equal to 1 .

\section{NumericAl RESUlts AND Discussion}

In this section, the theoretical analysis presented in the paper is verified via Monte Carlo simulations. In all experiments, we set $P_{S}=P_{R}=1$ and let the noise variances determine the SNR in the links of the relay network. In Fig. 2 theoretical and simulation results for the BER performance of simple MRC, thresholded MRC and link adaptive MRC are depicted for various $\bar{\gamma}_{S D}$ SNRs and $\bar{\gamma}_{S R}=\bar{\gamma}_{R D}=15 \mathrm{~dB}$. The theoretical curves have been obtained based on the BER expressions derived for the three schemes in Section III. We observe from Fig. 2 that theoretical and simulation results almost coincide, thus corroborating our analysis. Moreover, it can be noticed that link adaptive MRC outperforms the other two schemes for medium and high SNR values, while thresholded MRC is superior in a low SNR regime. In Figs. 3 and 4, the method proposed in Section IV is compared to link-adaptive MRC and the optimal threshold selection method of [1]. In both figures, $\bar{\gamma}_{R D}$ was taken equal to $15 \mathrm{~dB}$, while $\bar{\gamma}_{S R}=5 \mathrm{~dB}$ in Fig. 3 and $\bar{\gamma}_{S R}=15 \mathrm{~dB}$ in Fig. 4. Note that in [1], besides the optimal threshold, an approximate BER expression has been also derived. In our experiments the optimal threshold of [1] has been incorporated in the exact BER expression obtained for thresholded MRC in Section III. We see from Figs. 3 and

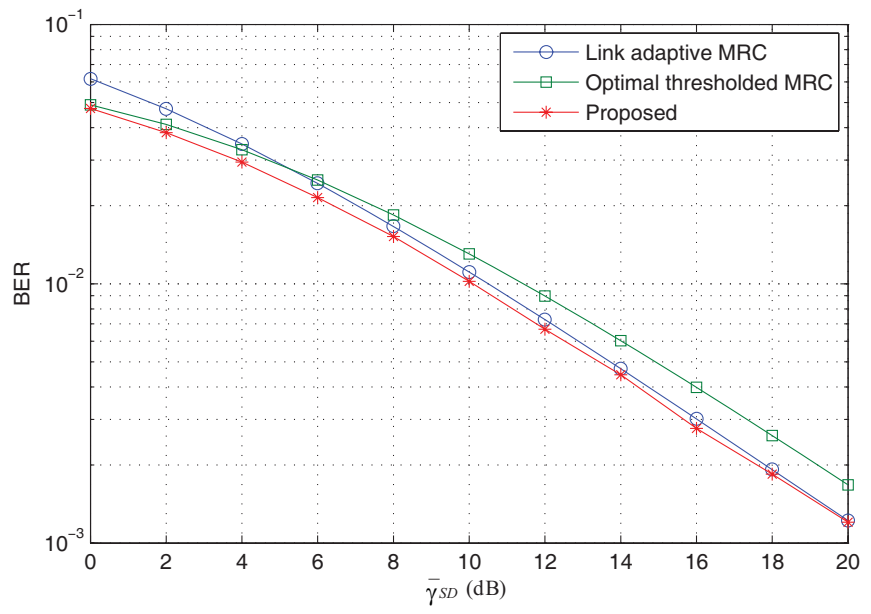

Fig. 3. Performance comparison of three DaF schemes for $\bar{\gamma}_{S R}=5 \mathrm{~dB}$ and $\bar{\gamma}_{R D}=15 \mathrm{~dB}$.

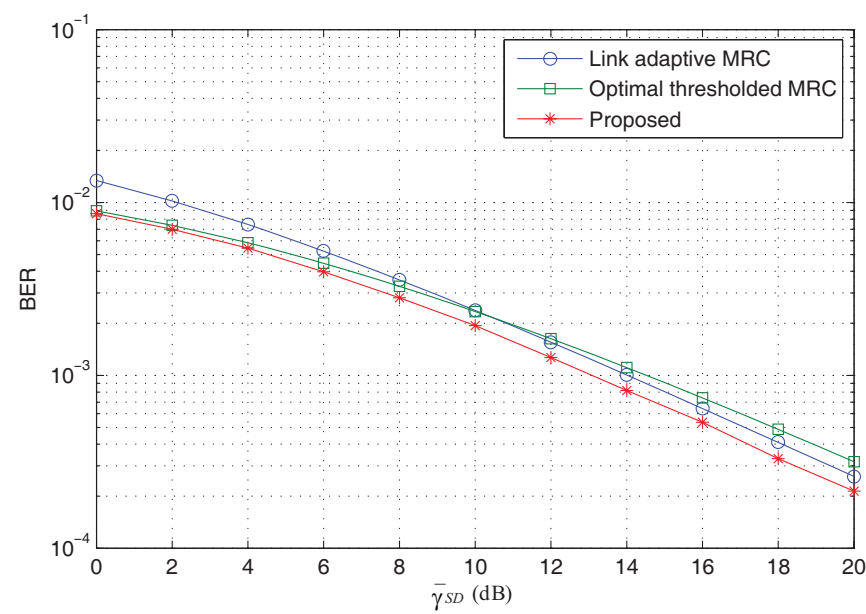

Fig. 4. Performance comparison of three $\mathrm{DaF}$ schemes for $\bar{\gamma}_{S R}=15 \mathrm{~dB}$ and $\bar{\gamma}_{R D}=15 \mathrm{~dB}$.

4 that the proposed method for selecting the weighting factor $w$ outperforms the other two relaying strategies, for all SNR values and for both low and medium reliability of the sourceto-relay link.

It should be mentioned that from experiments that follow the simulation settings used in [2] (and not reported here due to space limitations), it can be shown that the proposed relay strategy has diveristy order two, similar to the link adaptive MRC scheme described in [2].

\section{CONCLUSIONS}

A unified BER performance analysis of three DaF cooperative policies has been developed in this paper. Closed- 
form analytical BER expressions have been derived under a common framework. The main idea was to consider that the decisions at the relay are properly weighted depending on the reliability of the source-to-relay link. Based on this idea an optimal weighting factor selection method has been proposed, which, as verified by simulations, outperforms in terms of BER all previously known related schemes.

\section{APPENDIX}

The PDF of $d$ given in (18) equals the PDF of the RV $\sum_{i=1}^{8} \lambda_{i} u_{i}^{2}$, where $\lambda_{i}, i=1,2, \ldots, 8$ are the eigenvalues of the matrix $\mathbf{C}_{\mathbf{v}} \mathbf{A}$ and $u_{i}$ are independent, identically distributed standard normal RVs [9]. It can be shown that matrix $\mathbf{C}_{\mathbf{v}} \mathbf{A}$ has four distinct eigenvalues each with multiplicity equal to two. More specifically by denoting with $l_{1}, l_{2}, l_{3}, l_{4}$ the distinct eigenvalues of $\mathbf{C}_{\mathbf{v}} \mathbf{A}$, it can be shown that

$$
\begin{gathered}
l_{1}=\lambda_{1}=\lambda_{2}=\frac{P_{S} s \sigma_{S D}^{2}+\sqrt{P_{S}^{2} s^{2} \sigma_{S D}^{4}+P_{S} \sigma_{S D}^{2} \sigma_{D}^{2}}}{2} \\
l_{2}=\lambda_{3}=\lambda_{4}=\frac{w P_{R} \tilde{s} \sigma_{R D}^{2}+\sqrt{w^{2} P_{R}^{2} \tilde{s}^{2} \sigma_{R D}^{4}+w P_{R} \sigma_{R D}^{2} \sigma_{D}^{2}}}{2} \\
l_{3}=\lambda_{5}=\lambda_{6}=\frac{P_{S} s \sigma_{S D}^{2}-\sqrt{P_{S}^{2} s^{2} \sigma_{S D}^{4}+P_{S} \sigma_{S D}^{2} \sigma_{D}^{2}}}{2}
\end{gathered}
$$

and

$l_{4}=\lambda_{7}=\lambda_{8}=\frac{w P_{R} \tilde{s} \sigma_{R D}^{2}-\sqrt{w^{2} P_{R}^{2} \tilde{s}^{2} \sigma_{R D}^{4}+w P_{R} \sigma_{R D}^{2} \sigma_{D}^{2}}}{2}$.

Thus, the decision variable $d$ is rewritten as

$$
d=\sum_{i=1}^{2} l_{i}\left(u_{2 i-1}^{2}+u_{2 i}^{2}\right)-\sum_{i=1}^{2}\left|l_{i+2}\right|\left(u_{2(i+2)-1}^{2}+u_{2(i+2)}^{2}\right) \text {. }
$$

that is, $d$ is expressed as the difference of sums of weighted independent chi-squared RVs with two degrees of freedom. Thus, assuming that $l_{1} \neq l_{2}$ and $l_{3} \neq l_{4}$, it can be shown that the PDF of $d$ given $s, \tilde{s}$ and $w$ is expressed as $^{2}[10$, p. 135]

$$
\begin{aligned}
p_{d \mid s, \widetilde{s}, w}(z) & =\frac{1}{2\left(\left|l_{3}\right|-\left|l_{4}\right|\right)} \\
& \times\left(\exp \left(\frac{z}{2\left|l_{3}\right|}\right) \frac{l_{3}^{2}}{\left(l_{1}+\left|l_{3}\right|\right)\left(l_{2}+\left|l_{3}\right|\right)}\right. \\
& \left.-\exp \left(\frac{z}{2\left|l_{4}\right|}\right) \frac{l_{4}^{2}}{\left(l_{1}+\left|l_{4}\right|\right)\left(l_{2}+\left|l_{4}\right|\right)}\right)
\end{aligned}
$$

for $z \leq 0$. Moreover, by integrating the PDF, the CDF of $d$ at zero is written as

$$
\begin{aligned}
\operatorname{Pr}(d \leq 0 \mid s, \tilde{s}, w) & =\frac{1}{\left(\left|l_{3}\right|-\left|l_{4}\right|\right)}\left(\frac{\left|l_{3}\right|^{3}}{\left(l_{1}+\left|l_{3}\right|\right)\left(l_{2}+\left|l_{3}\right|\right)}\right. \\
& \left.-\frac{\left|l_{4}\right|^{3}}{\left(l_{1}+\left|l_{4}\right|\right)\left(l_{2}+\left|l_{4}\right|\right)}\right)
\end{aligned}
$$

After substituting $l_{i}, i=1,2,3,4$ from (38)-(41), in (44), we end up with (20) and (21).

\footnotetext{
${ }^{2}$ Note that using a similar approach BER expressions can also be derived for the special cases $l_{1}=l_{2}$ and/or $l_{3}=l_{4}$.
}

\section{REFERENCES}

[1] F. A. Onat, A. Adinoyi, Y. Fan, H.Yanikomeroglu, J. S. Thompson, and I. D. Marsland, "Threshold selection for SNR-based selective digital relaying in cooperative wireless networks," IEEE Trans. Wireless Commun., vol. 7, no. 11, pp. 4226-4237, Nov. 2008.

[2] T. Wang, G. B. Giannakis, and R. Wang, "Smart regenerative relays for link-adaptive cooperative communications," IEEE Trans. Commun., vol. 56, no. 11, pp. 1950-1960, Nov. 2008.

[3] J. Boyer, D. Falconer, and H. Yanikomeroglu, "Multihop diversity in wireless relaying channels," IEEE Trans. Commun., vol. 52, no. 10, pp. 1820-2830, Oct. 2004.

[4] J. N. Laneman, D. Tse, and G. Wornell, "Cooperative diversity in wireless networks: efficient protocols and outage behavior," IEEE Trans. Inf. Theory, vol. 50, no. 12, pp. 3062-3080, Dec. 2004.

[5] T. Wang, A. Cano, G. B. Giannakis, and J. N. Laneman, "Highperformance cooperative demodulation with decode-and-forward relays," IEEE Trans. Commun., vol. 55, no. 7, pp. 1427-1438, July 2007.

[6] G. Scutari and S. Barbarossa, "Distributed space-time coding for regenerative relay networks," IEEE Trans. Wireless Commun., vol. 4, no. 5, pp. 2387-2399, Sep. 2005.

[7] A. Sendonaris, E. Erkip, and B. Aazhang, "User cooperation diversitypart II: implementation aspects and performance analysis," IEEE Trans. Commun., vol. 51, no. 11, pp. 1939-1948, Nov. 2003.

[8] M. K. Simon and M.-S. Alouini, Digital Communication over Fading Channels. John Wiley and Sons, 2005.

[9] G. A. Ropokis, A. A. Rontogiannis, and P. T. Mathiopoulos, "Quadratic forms in normal RVs: theory and applications to OSTBC over Hoyt fading channels," IEEE Trans. Wireless Commun., vol. 7, no. 12, pp. 5009-5019, Dec. 2008.

[10] A. M. Mathai and S. B. Provost, Quadratic Forms in Random Variables: Theory and Applications. Marcel Dekker, Inc, 1992. 